$\checkmark$ Research Square

\title{
Unilateral tongue mass after iatrogenic lingual nerve injury: A case report
}

Hyung Chae Yang

Chonnam National University Medical School

Min-Keun Song ( $\nabla$ drsongmk@daum.net)

Chonnam National University Hospital https://orcid.org/0000-0001-8186-5345

\section{Case report}

Keywords: Tongue, Hemangioma, Lingual nerve injury, latrogenic disease, Submandibular gland

Posted Date: July 22nd, 2020

DOl: https://doi.org/10.21203/rs.3.rs-47198/v1

License: (9) This work is licensed under a Creative Commons Attribution 4.0 International License. Read Full License 


\section{Abstract}

Backgroud: This is the first report of hemangioma as a long-term complication of iatrogenic lingual nerve injury during submandibular gland extirpation. Case presentation: A 69-year-old woman presented with a tongue mass, which had grown slowly over 20 years. Submandibular gland (SMG) extirpation performed 40 years prior. MRI and CT revealed evidence of SMG extirpation. In addition, MRI and CT also revealed several enhancing lesions (hemangiomas) in the right sublingual space, as well as in the right anterior part of the tongue. An electrodiagnostic study showed right-sided lingual neuropathy. Psychosensory taste test showed taste disturbance in the affected side tongue. Conclusions: It should be considered to the lingual nerve preservation during the sublingual gland extirpation. It might cause the secondary traumatic pathology.

\section{Background}

Latrogenic lingual nerve injury is a severe complication of submandibular gland (SMG) extirpation. The lingual nerve innervates the anterior two-thirds of the tongue. Patients with lingual nerve injury lack sensory function in that part of the tongue, so may be unaware of serious injuries therein caused by tongue bites, for example. However, little is known regarding the long-term consequences of the sensory deficits caused by iatrogenic lingual nerve injury. Here, we describe a patient who experienced long-term sensory deficits following iatrogenic lingual nerve injury.

\section{Case Presentation}

A 69-year-old woman presented with a tongue mass. Physical examination revealed multiple red-tobluish-colored tortuous soft masses on the right side of the tongue. The midline of the tongue tip was shifted to the left due to the mass (Fig. 1A and 1B). The patient had a surgical scar in the right submandibular area due to SMG extirpation performed 40 years prior. Following that surgery, she lost all sensation on the right side of her tongue, and had repeatedly bitten and damaged that side. The tongue mass had grown slowly over 20 years.

Magnetic resonance imaging revealed evidence of SMG extirpation. In addition, there were several enhancing lesions in the right sublingual space, as well as in the right anterior part of the tongue, with no obvious invasion of the adjacent mandible (Fig. 2). Computed tomography revealed heterogeneously enhancing masses with internal calcification (phlebolith).

An electrodiagnostic study was performed to assess the sensory nerve action potential of the lingual nerve. Peak latencies were observed at 1.30 and $1.98 \mathrm{~ms}$ in the right and left lingual nerves, respectively; the respective amplitudes were 20.9 and $2.1 \mathrm{mV}$. The amplitude of the sensory nerve action potential in the right lingual nerve was markedly reduced, which may have contributed to the iatrogenic lingual nerve injury. The patient's sense of taste was evaluated using sucrose (sweet), $\mathrm{NaCl}$ (salty), citric acid (sour), and quinine $\mathrm{HCl}$ (bitter). The patient could not detect any salty or bitter tastes, and had reduced sensitivity 
to sweet and sour tastes relative to the other side of the tongue. Therefore, a clinical diagnosis of hemangioma due to iatrogenic lingual nerve injury was made.

We planned surgical removal of hemangioma due to dysarthria and the risk of massive bleeding. However, the patient refused surgery; instead, she was educated regarding oromotor exercises to prevent tongue biting. No further growth of hemangioma was observed during 2 years of follow-up.

\section{Discussion And Conclusion}

This patient had unilateral tongue hemangioma caused by a long-term sensory deficit, in turn due to iatrogenic lingual nerve injury that occurred during SMG extirpation. To the best of our knowledge, this is the first report of hemangioma as a long-term complication of iatrogenic lingual nerve injury occurring during SMG extirpation, and provides additional evidence of the importance of avoiding iatrogenic lingual nerve injury during surgery.

Generally, hemangiomas present as deep red-colored, soft, smooth or lobulated, and sessile or pedunculated masses of various sizes that may blanch on touch pressure. ${ }^{1-3}$ Various conditions may contribute to the formation of hemangioma. ${ }^{3}$ Trauma can also cause hemangioma. ${ }^{4}$ Recurrent tongue bite due to a sensory deficit may have been the source of traumatic injury in this patient.

We performed an electrodiagnostic study to evaluate lingual nerve injury. The reduced sensory nerve action potential amplitudes on the right side of the tongue denoted partial lingual neuropathy, particularly involving the trigeminal division. An injury mechanism involving severance, followed by regeneration, of the lingual nerve was suspected, and may have been revealed by electrodiagnostic study of the trigeminal nerve. However, the chorda tympani nerve cannot be evaluated by an electrodiagnostic study. Therefore, we used a taste test, which revealed the loss of salty and bitter taste on the right side of the tongue.

Most hemangiomas are asymptomatic and disappear without treatment. However, the patient in this case exhibited dysarthria, and the hemangioma had grown slowly over an extended period. Therefore, we planned excision of the hemangioma due to dysarthria and the risk of massive bleeding, but the patient preferred conservative treatment. She was referred to the Department of Rehabilitation Medicine and was educated regarding oromotor exercises to prevent tongue bites. No further growth of hemangioma was observed during 24 months of follow-up.

\section{Abbreviations}

SMG ; submandibular gland

\section{Declarations}

\section{Ethical Approval and Consent to participate}


This study was approved by the Institutional Review Board of Chonnam National University Hospital (CNUH-EXP-2019-190).

\section{Consent for publication}

We received a written informed consent for the publication from the patient.

\section{Availability of supporting data}

The datasets used during the current study are available from the corresponding author on reasonable request. 2

\section{Competing interests}

The authors declare that they have no competing interests.

\section{Funding}

This research was supported by the Bio \& Medical Technology Development Program of National Research Foundation of Korea (NRF) funded by the Korean government MSIP (NRF2017M3A9E8023021), and a grant from Chonnam National University Hospital Biomedical Research Institute (BCRI 18020).

\section{Author contributions}

Investigation, Resources: Hyung Chae Yang, Min-Keun Song

Writing - original draft: Hyung Chae Yang

Writing - review \& editing: Min-Keun Song.

\section{Acknowledgements}

Not applicable

\section{Authors' information}

First author: Hyung Chae Yang, MD \& PhD.

Assistant professor

Department of Otolaryngology-Head and Neck Surgery,

Chonnam National University Medical School and Chonnam National University Hospital 
Corresponding author : Min-Keun Song, MD \& PhD

Clinical assistant professor

Department of Physical \& rehabilitation medicine

Chonnam National University Hospital \& Medical School

\section{References}

1. Kamala KA, Ashok L, Sujatha GP. Cavernous hemangioma of the tongue: A rare case report. Contemp Clin Dent. 2014;5(1):95-98.

2. Werner JA, Dunne AA, Folz BJ, et al. Current concepts in the classification, diagnosis and treatment of hemangiomas and vascular malformations of the head and neck. Eur Arch Otorhinolaryngol. 2001;258(3):141-149.

3. Kripal K, Rajan S, Ropak B, Jayanti I. Cavernous hemangioma of the tongue. Case Reports in Dentistry. 2013;2013:3.

4. Richter GT, Friedman AB. Hemangiomas and vascular malformations: current theory and management. Int J Pediatr. 2012;2012:10.

\section{Figures}
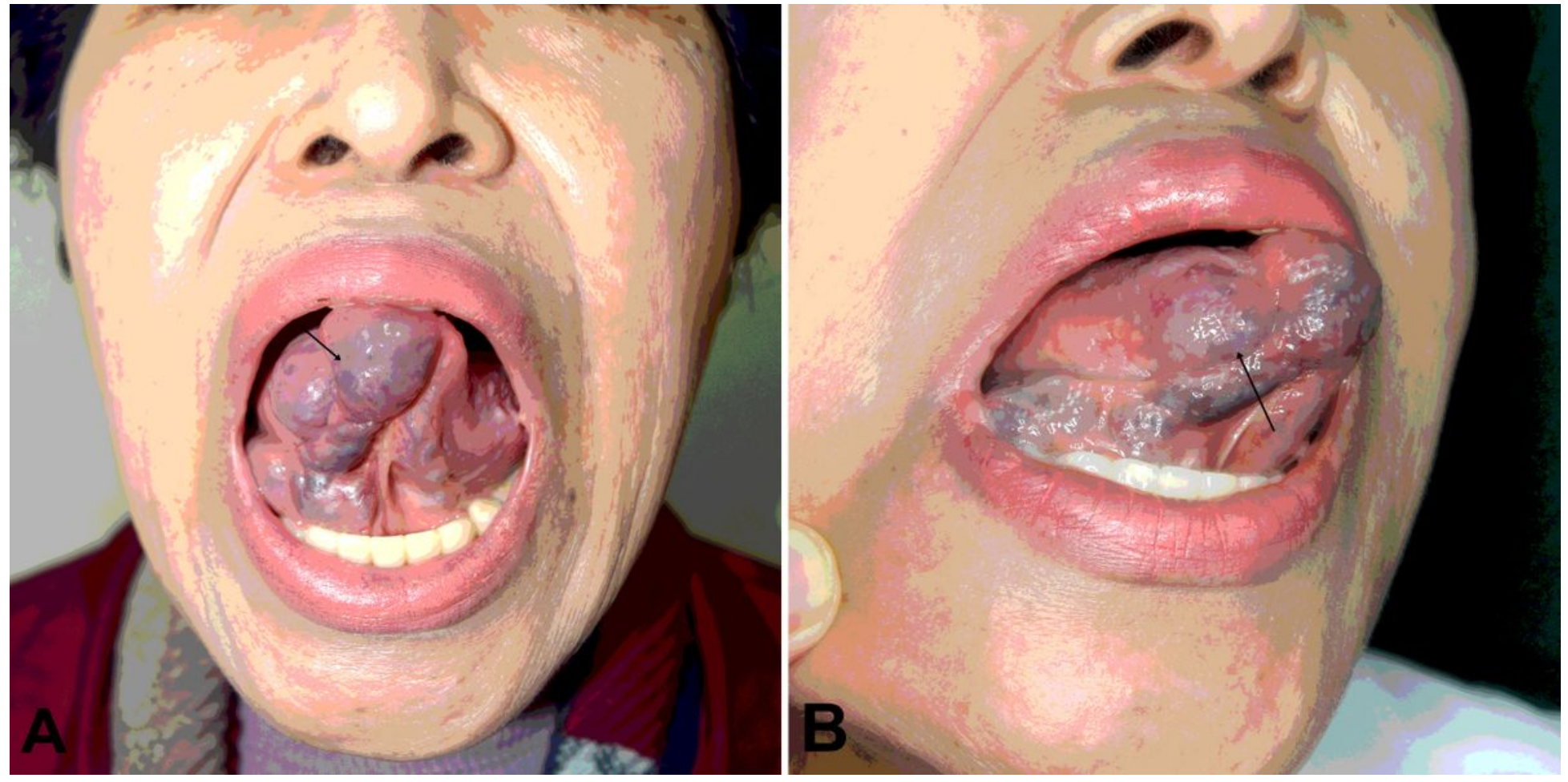

Figure 1 
A. Hemangioma on the right side of the tongue. Multiple red-to-bluish-colored tortuous soft masses on the right side of the tongue. The patient had undergone submandibular gland extirpation (SMG) 40 years prior. B. Hemangioma on the right side of the tongue. Multiple red-to-bluish-colored tortuous soft masses on the right side of the tongue. The patient had undergone submandibular gland extirpation (SMG) 40 years prior.
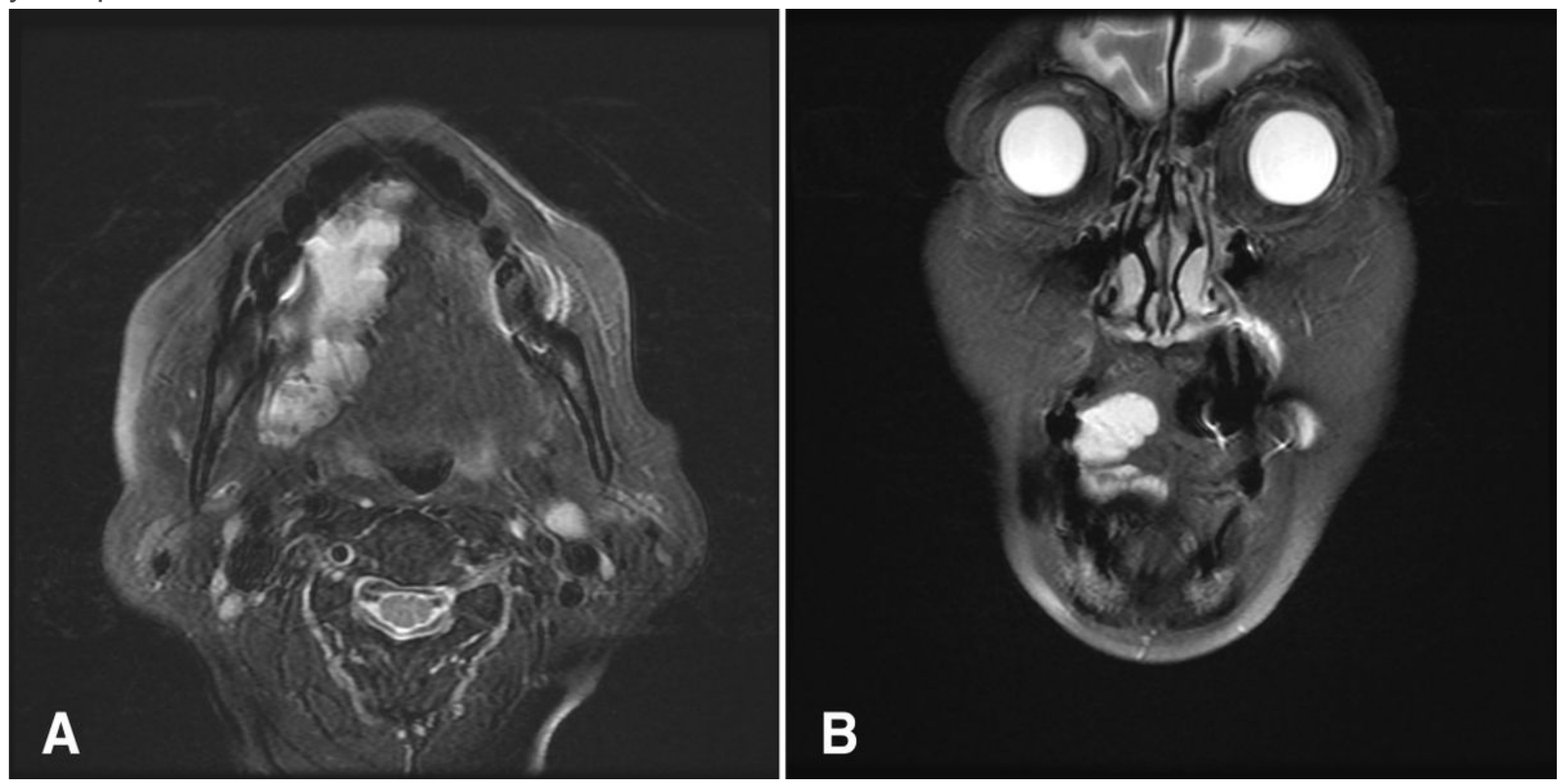

Figure 2

A. Magnetic resonance images of unilateral tongue hemangioma. The conglomerate enhancing masses with high intensity in T2-weighted images are confined to the right side of the tongue in the coronal view (A) and sagittal view (B). B. Magnetic resonance images of unilateral tongue hemangioma. The conglomerate enhancing masses with high intensity in T2-weighted images are confined to the right side of the tongue in the coronal view (A) and sagittal view (B). 